\title{
AN EFFICIENT ORDER REDUCTION STRATEGY IN EARTHQUAKE NONLINEAR RESPONSE ANALYSIS OF STRUCTURES
}

\author{
F. Bamer ${ }^{1}$, A. Kazemi Amiri ${ }^{2}$ and C. Bucher ${ }^{3}$ \\ ${ }^{1}$ RWTH-Aachen University, Institute of General Mechanics, \\ Templergraben 64, 52062 Aachen, Germany \\ e-mail: bamer@iam.rwth-aachen.de \\ 2 Vienna Doctoral Programme on Water Resource Systems, \\ TU Vienna, Karlsplatz 13/222, 1040 Vienna, Austria \\ e-mail: mehrad_hoo@yahoo.com \\ ${ }^{3}$ Center for Mechanics and Structural Dynamics, \\ TU Vienna, Karlsplatz 13/206, 1040 Vienna, Austria \\ e-mail: Christian.Bucher@tuwien.ac.at
}

Keywords: Model order reduction, Proper orthogonal decomposition, Friction pendulum system

\begin{abstract}
Since earthquake dynamic response analysis of large and complex structures are computationally time demanding, efficient methods that can reduce the system order are of high interest. In this sense, there are different methods available, which try to provide a proper equivalent model. However, in the presence of nonlinearities in the structural elements, most of those methods are ruled out due to their linear assumptions. Therefore, this contribution aims at providing an efficient strategy, which can reduce the order of the nonlinear structural model while retaining important structural characteristics for further earthquake dynamic response analysis. The model order reduction (MOR) strategy is developed based on the proper orthogonal decomposition (POD) method to derive a set of nonlinear deterministic POD modes according to the information of the response history (snapshots) of the full order structure under one or a set of representative earthquake excitations. Subsequently, the POD modes are utilized to create the reduced-order models of the structure subjected to different earthquake excitations. Then, the reduced order models need substantially less amount of computational time in comparison to the full order models. This study presents the application results of the introduced new strategy to a realistic building structure, which is base-isolated by means of frictional bearing elements for better seismic performance. The results demonstrate accurate approximations of the physical (full) responses by means of this new MOR strategy if the probable behavior of the structure has already been captured in the POD snapshots.
\end{abstract}




\section{INTRODUCTION}

The evaluation of the response history of a structure in the time domain is one of the main topics in earthquake engineering and structural dynamics. Often it is common practice to create simple structural models, e.g. multistory shear frames, which should be able to describe the structural behavior and peculiarities of the real structure. This approach leads to useful results for the investigation of rather simple and uniform structures in order to draw meaningful engineering decisions regarding structural resistance. On the contrary, the analysis of complicated systems can require the application of nonlinear high-order systems, as a characterization by a low dimensional structural model could lead to an oversimplification, i.e. important motion patterns could be ignored. Therefore, an effective strategy is to obtain a set of a low number of "important" equations of motion that approximates the high-dimensional nonlinear dynamical system as accurately as possible, that is, model order reduction (MOR).

The solution of the nonlinear set of equations of motion in the time domain is realized by numerical algorithms, which require computational effort if the number of DOF is high. Even the response calculation of linear systems can be expensive, as a factorization of the stiffness matrix is necessary to solve the eigenvalue problem and calculate the natural modes of vibration.

An alternative is to replace a high-dimensional nonlinear set of equations of motion by a reduced set, providing the main dynamic behavior of the system to reach the required level of accuracy. MOR methods are used in many fields of research, where high-dimensional systems are dealt. Some review papers of MOR, especially for structural dynamic applications, are presented by Rega and Troga [3] and Koutsovasilis and Beitelschmidt [4] as well as the books of $\mathrm{Qu}$ [2] and Schilders et al. [5]. The classical but also effective method of modal truncation is well-known in the field of earthquake engineering, which is however mainly applicable to linear systems.

This paper concentrates on a new MOR strategy based on the proper orthogonal decomposition (POD) method. The POD provides a low dimensional uncorrelated description (basis vectors), by which a high-dimensional correlated process, e.g. structural response, is spanned. Firstly, it was used as a statistical formulation in the papers of Kosambi [6], Karhunen [7] and Loeve [8]. Following this mathematical basis of the POD, which is also known as KarhunenLoeve Decomposition and Principal Component Analysis, was applied in many fields of research, including turbulence and coherent structures, wind engineering, image processing and structural dynamics.

The first paper regarding the field of structural dynamics was written by Cusumano et al. [9] in the early 1990's, who presented an experimental study of dimensionality in an elastic impact oscillator. In the papers [10] and [11] of Feeny and Kappagantu a relation of the proper orthogonal modes to normal modes of vibration is investigated. Then they used the POD as they so call optimal modal reduction and exploit the benefits of the application of these modes in comparison to the linear natural modes. Furthermore, Kappagantu and Feeny investigated in [12] and [13] the dynamics of an experimental frictionally excited beam and they verified that the proper orthogonal modes are efficient in capturing the dynamics of the system. Liang et. al. [14] discuss the realizations of the POD, i.e. Karhunen-Loeve Decomposition, principal component analysis and singular value decomposition and compare these three methods. Kerschen and Golivani [15] analyze the physical interpretation of the POD modes and its relation to the singular value decomposition and in [16] they investigated POD based on auto-associative neural networks.

The necessity to describe a high dimensional set by a small set of equations of motion, i.e. 
MOR, has aroused interests mainly in the last two decades in the field of earthquake engineering, some papers related to this issue are e.g. [27] and [28]. More specified to the related issue Tubino et. al. [17] investigated the seismic ground motion of the support points of a structure and denominate the POD as a very efficient tool to simulate multi-variate processes. Bucher [32] examined the stabilization of explicit time integration methods for analysis of nonlinear structural dynamics by modal reduction. Gutierrez and Zaldivar investigated in [30] how to handle the stability problem of explicit time integration by modal truncation methods more related to problems in earthquake engineering and structural dynamics and following in [29], they applied the Karhunen-Loeve Decomposition, which is formally identical to the POD analysis, to capture the essential characteristics of nonlinear systems and provide experimental examples conducted on a shaker table. Bamer and Bucher [18] developed a MOR strategy applying the POD method for transient excited structures resting on one-dimensional friction elements. This study presented a powerful combination of the POD and explicit time integration schemes.

The current work investigates the extension of the POD-based MOR strategy, which is applicable to nonlinear systems in contrast to the method of modal truncation. The new strategy pursues the following objective: a low number of deterministic nonlinear modes (i.e. set of POD modes) is determined that defines a representative characterization of the structural behavior. Therefore, due to the information content of the full or a part of the time response of the structure to one representative excitation a set of deterministic modes, i.e. POD modes, is evaluated. Subsequently, this set of modes is utilized to project the equations of motion of a structure under different earthquake excitations onto POD coordinates and following an order truncation is performed in a similar manner as the application of modal truncation to linear systems.

It is attached importance to demonstrate this new strategy and its advantages on a practical application. The method is applied to the dynamic model of a realistic building structure. The building is erected on friction pendulum bearings for the sake of seismic isolation to minimize the transferred acceleration to the building during an earthquake. A three-dimensional dynamic model of the base isolated structure is derived by implementing the finite element model of the structure and the bi-directional friction pendulum systems. The subsequent section deals with the nonlinear dynamic model of the base-isolated structure. Following, the new POD-based MOR strategy and the example of its practical application is provided. Finally, the discussion on the results and conclusions are given.

\section{Nonlinear model order reduction}

The $n$-dimensional set of equations of motion of a structure with nonlinear material behavior excited by horizontal components of ground acceleration is expressed as (cf. Chopra [20])

$$
\mathbf{M} \ddot{\mathbf{x}}+\mathbf{C} \dot{\mathbf{x}}+\mathbf{R}(\mathbf{x})=-\mathbf{M}\left(\mathbf{f}_{\mathbf{x}} \ddot{x}_{g}+\mathbf{f}_{\mathbf{y}} \ddot{y}_{g}\right),
$$

where $\mathbf{M}$ and $\mathbf{C}$ are mass- and damping square matrices of order $n$ and $\mathbf{R}(\mathbf{x})$ is the nonlinear internal restoring force vector dependent on the displacement $\mathbf{x}$ with the dimension $n \times 1$. The right hand side of the set of equations of motion describes the earthquake excitation term, while $\ddot{x}_{g}$ and $\ddot{y}_{g}$ denote the ground acceleration in $x$ - and $y$-direction and $\mathbf{f}_{j},(j=x, y)$ are the influence vectors in the corresponding direction. It is

$$
\mathbf{f}_{\mathbf{x}}\left(x_{i}\right)=1, \quad \mathbf{f}_{\mathbf{y}}\left(y_{i}\right)=1, \quad i=1 \ldots n,
$$

at the global $x$ and $y$ degrees of freedom of all nodes, whereas the other components of $\mathbf{f}_{\mathbf{x}}$ and $\mathbf{f}_{\mathbf{y}}$ are zero. Thus, $i$ describes the number of nodes of the FE discretized structure. This general 
approach indicates that in this paper the ground acceleration in the corresponding direction, i.e. $x$-, $y$ - or $z$-direction, is equal in all structural support points. In the following equations the term on the right hand side of the set of equations of motion (1) is denominated by $\mathbf{F}(t)$, which has the unit of a force.

Nonlinear systems, as they are depicted in Equation (1), have generally to be solved by the application of a numerical algorithm, that is, a step by step procedure in the time domain in order to obtain the response of the structure as a function of time. The necessity of application of a numerical method inevitably leads to the existence of computational effort if $n$ is a large number. Therefore, the approximation by a low, dimensional description of the system seems to be useful, i.e. the application of MOR.

The main goal of MOR techniques is primarily to define a transformation matrix $\mathbf{T} \in$ $\mathbb{R}^{n \times m}, m \ll n$ to approximate the displacement vector $\mathbf{x} \in \mathbb{R}^{n}$ through a reduced coordinate vector $\mathbf{q}_{\mathbf{r}} \in \mathbb{R}^{m}$ by the relation (cf. Koutsovasilis and Beitelschmidt [19])

$$
\mathrm{x}=\mathbf{T} \mathbf{q}_{\mathrm{r}}
$$

such that the dynamic properties of the system are preserved and the error is small. The notation of the variable $m \in \mathbb{N}$ is the dimension of the reduced subspace.

The projection of the nonlinear system defined by Equation (1) onto that subspace leads to another second-order ordinary differential equation (cf. Koutsovasilis and Beitelschmidt [19])

$$
\mathbf{m}_{\mathbf{r}} \ddot{\mathbf{q}}_{\mathbf{r}}+\mathbf{c}_{\mathbf{r}} \dot{\mathbf{q}}_{\mathbf{r}}+\mathbf{r}=\mathbf{f}_{\mathrm{r}}
$$

where $\mathbf{m}_{\mathbf{r}}=\mathbf{T}^{\mathbf{T}} \mathbf{M T}, \quad \mathbf{c}_{\mathbf{r}}=\mathbf{T}^{\mathbf{T}} \mathbf{C T} \in \mathbb{R}^{m \times m}$ are mass- and damping matrix and $\mathbf{f}_{\mathbf{r}}=$ $\mathbf{T}^{\mathbf{T}} \mathbf{F}(t) \in \mathbb{R}^{m \times 1}$ is the force vector in the reduced subspace. It should be noted that the reduced system matrices $\mathbf{m}_{\mathbf{r}}$ and $\mathbf{c}_{\mathbf{r}}$ are generally not diagonal. The vector of the restoring forces in the reduced subspace is

$$
\mathbf{r}=\mathbf{T}^{\mathbf{T}} \mathbf{R}(\mathbf{x})=\mathbf{T}^{\mathbf{T}} \mathbf{R}\left(\mathbf{T} \mathbf{q}_{\mathbf{r}}\right) .
$$

Consequently, one necessity of nonlinear MOR is the evaluation of the vector of the restoring forces in the physical (full) coordinate at every time step.

\section{The proper orthogonal decomposition and nonlinear modes}

Modal truncation is a widely-used tool and an effective method for order reduction of linear systems in the field of earthquake engineering. An accurate approximation of the response history is achieved by applying a small number of lower structural modes proportional to the number of degrees of freedom. In this work, the objective is to find a new strategy that is applicable to nonlinear systems in a similar manner to modal truncation. The approach is to define a set of deterministic modes that can be evaluated from the information of an existing response history of the structure. Consequently, this set of modes contains nonlinear motion patterns if the structure shows nonlinear response behavior to the excitation.

The proposed strategy is established based on the theory of the POD method. Generally, the POD ([2], , [14], [21], [22], [23], [24]) is a straightforward approach to obtain a low-dimensional uncorrelated process from a correlated high dimensional or even infinite-dimensional process. Holmes et al. [24] examined the theoretical background of the POD and its properties profoundly. In the following, the mathematical basics of the POD are discussed shortly, but as the paper is more targeted to the strategic approach in earthquake engineering, the dealing of the mathematical background and the numerical problems are limited to an essential minimum. 
The aim of the POD is to find a set of ordered orthonormal basis vectors in a subspace so that samples in a sample space are expanded in terms of $l$ basis vectors in an optimal form. This means that the POD is able to find an orthonormal basis, which describes an observation vector in a subspace better than any other orthonormal basis can do. A measure for this problem is the mean square error (cf. Qu [2])

$$
E\left\{\|\mathbf{x}-\mathbf{x}(l)\|^{2}\right\} \leq E\left\{\|\mathbf{x}-\hat{\mathbf{x}}(l)\|^{2}\right\}
$$

where $\mathbf{x} \in \mathbb{R}^{n \times 1}$ is the random vector, $\mathbf{x}(l)$ is the approximation of this random vector in an $l$-dimensional POD subspace and $\hat{\mathbf{x}}(l)$ is the approximation of the random vector by any other possible orthonormal basis. Therefore, the random vector can be expressed as (cf. Qu [2])

$$
\mathbf{x}=\boldsymbol{\Phi}_{\mathbf{p}} \mathbf{q}_{\mathbf{p}}, \quad \boldsymbol{\Phi}_{\mathbf{p}}=\left[\varphi_{\mathbf{p}, \mathbf{1}}, \varphi_{\mathbf{p}, \mathbf{2}}, \ldots, \varphi_{\mathbf{p}, \mathbf{s}}\right] \quad \text { and } \quad \mathbf{q}_{\mathbf{p}}=\left[q_{p, 1}, q_{p, 2}, \ldots, q_{p, s}\right],
$$

where $\varphi_{\mathbf{p}, \mathbf{i}}$ are the POD modes and $q_{p, i}$ denote the coordinates in the POD subspace and $s$ is the number of realizations of the random vector (also called snapshots). This leads to an optimization problem with the following objective function (cf. Qu [2])

$$
\epsilon^{2}(l, t)=E\left\{\|\mathbf{x}-\mathbf{x}(l)\|^{2}\right\} \rightarrow \min
$$

subject to the orthonormality condition (cf. Qu [2])

$$
\varphi_{\mathbf{p}, \mathbf{i}}^{\mathbf{T}} \varphi_{\mathbf{p}, \mathbf{j}}=\delta_{i j}(i, j=1,2, \ldots, s) .
$$

The transformation into the $l$-dimensional POD subspace is a truncation of the first $l$ lower POD modes (cf. Qu [2])

$$
\mathbf{x}(l) \approx \Phi_{\mathbf{p}} \mathbf{q}_{\mathbf{p}}, \quad \Phi_{\mathbf{p}}=\left[\varphi_{\mathbf{p}, \mathbf{1}}, \varphi_{\mathbf{p}, \mathbf{2}}, \ldots, \varphi_{\mathbf{p}, \mathbf{1}}\right], \quad l<s \ll n .
$$

In structural dynamics, systems are discretized in space and time and the random vector is realized by $s$ observations at different time instances (cf. Han and Feeny [25])

$$
\mathbf{X}_{\mathbf{s}}=\left[\mathbf{x}_{\mathbf{t}_{1}}, \mathbf{x}_{\mathbf{t}_{2}}, \ldots, \mathbf{x}_{\mathbf{t}_{\mathbf{s}}}\right]=\left(\begin{array}{ccc}
x_{1}\left(t_{1}\right) & \cdots & x_{1}\left(t_{s}\right) \\
\cdots & \cdots & \cdots \\
x_{n}\left(t_{1}\right) & \cdots & x_{n}\left(t_{s}\right)
\end{array}\right) .
$$

These observation vectors $\mathbf{x}_{\mathbf{t}_{\mathbf{i}}}$ are called snapshots and, therefore, in the literature often the observation matrix $\mathbf{X}_{\mathbf{s}}$ is called snapshot matrix containing $s$ snapshots (observations). $\mathbf{x}_{\mathbf{t}_{\mathbf{i}}}$ can be measurements or solution vectors of a dynamical system at different time instances ([21]). If $\boldsymbol{\mu}$ is the expectation of all observations, then the sample covariance matrix $\boldsymbol{\Sigma}_{\mathrm{s}}$ of this random vector, which is realized by the observation matrix, is defined by (cf. Kerschen et. al. [26])

$$
\boldsymbol{\Sigma}_{\mathbf{s}}=E\left\{(\mathbf{x}-\boldsymbol{\mu})^{T}(\mathbf{x}-\boldsymbol{\mu})\right\} .
$$

The POD modes and the POD values are defined by the eigensolution of the sample covariance matrix. If the data have zero mean, the covariance matrix is (cf. Kerschen et al. [26])

$$
\Sigma_{\mathbf{s}}=\frac{1}{s} \mathbf{X}_{\mathbf{s}}^{T} \mathbf{X}_{\mathbf{s}}
$$

and the POD is realized by the singular value deStructurescomposition (SVD) of the observation matrix $\mathbf{X}_{\mathbf{s}}$. The POD modes $\varphi_{\mathbf{p}, \mathbf{i}}$ are equal to the left singular vectors and the POD values $\lambda_{p, i}$ to 


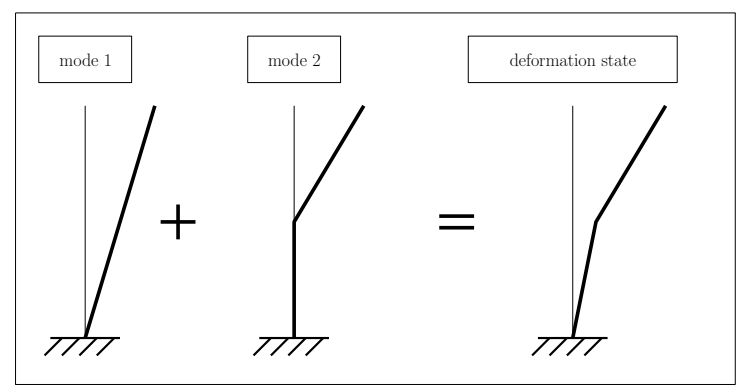

Figure 1: Appropriate mode superposition

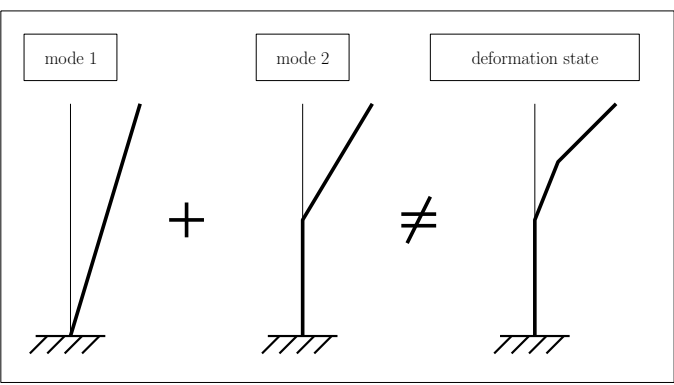

Figure 2: Inappropriate mode superposition

the singular values of $\mathbf{X}_{\mathbf{s}}$, which are all real and positive and arranged in a rectangular diagonal matrix in descending order. The energy, which is contained by the snapshot matrix, is defined by the summation of the POD values, i.e. $V=\sum_{i=0}^{s} \lambda_{p, i}$. As a consequence, the energy ratio of the $i_{t h}$ POD mode is (cf. Kerschen et al. [26])

$$
V_{i}=\frac{\lambda_{p, i}}{\sum_{i=0}^{s} \lambda_{p, i}}
$$

In structural dynamics applications the sum of only a few POD values often captures 99.99 percent of the total energy included in the observation matrix, which reflects the big advantage of the POD, i.e. the property of optimality with respect to energy in a least square sense.

Practically, nonlinear effects must be sufficiently captured in the snapshot matrix (11) derived from the representative earthquake exciStructurestation. This is in order to create the capability of providing the possible nonlinear responses that can appear in the response history of the structure excited by another earthquake event. Consequently, nonlinear effects that are not contained in the snapshot matrix, such as plastic hinges or nonlinear sliding of a friction isolator cannot be displayed in the system response. This issue is depicted qualitatively in Figures 1 and 2. Two fictitious deformation states of a simple surrogate model are shown. Obviously, the two deterministic modes in Figure 1 are sufficient in order to represent the deformation state of the model. On the contrary, the set of deterministic modes is insufficient to represent the deformation state in Figure 2 and, consequently, the existing set of modes must be expanded by an additional mode in order to ensure a representation of the correct deformation state. Related to this paper, the conclusion is to capture nonlinear structural reactions in the snapshot matrix in order to provide accurate approximations of the structural response excited by different earthquake events.

Structures

\section{The new strategy}

In this strategy, a snapshot matrix, which contains information about the response of the structure including nonlinear actions, must be found. Therefore, the set of full-order equations of motion of a structure is integrated numerically over the time history of the whole or a part of a representative earthquake excitation and the snapshots are selected from a chosen amount of response vectors, i.e. the displacement vectors at different time instances. Evaluation of the snapshot matrix comprises the time-consuming part of this MOR approach, therefore, the most customary and time-saving approach is to integrate over a small time window in the beginning of a representative earthquake excitation. Nevertheless, in order to increase the probability of recording sufficient linear and nonlinear response patterns, it seems to be reasonable to spread 


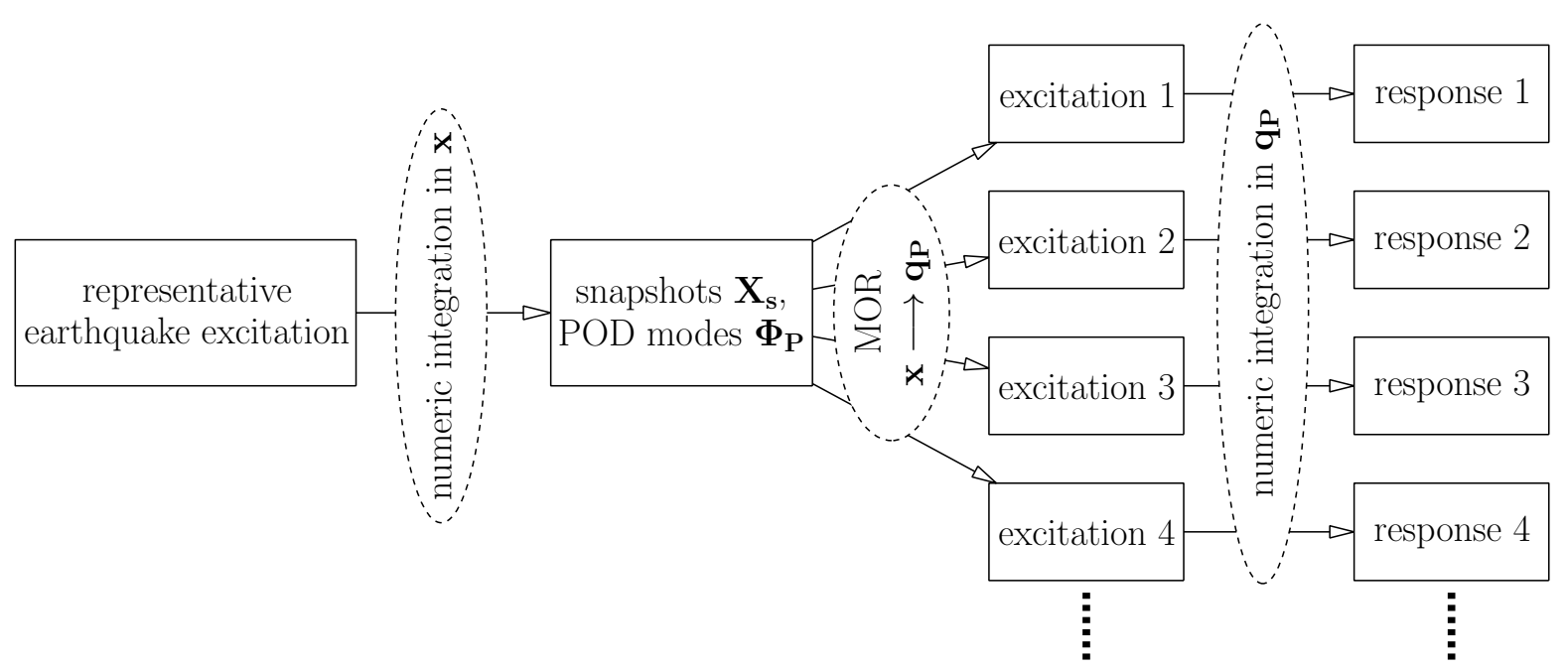

Figure 3: Approach of the new strategy

the snapshot records in equidistant time instances over the whole time history of the earthquake response. Additionally, it is of highest advantage to evaluate the full solutions to more than one earthquake event and spread the snapshots over all of the response histories. This procedure increases the probability of capturing all necessary motion patterns in order to provide an accurate representation by the reduced set of equations. Different earthquake excitations can affect nonlinearities, such as plastic hinges, at different parts of the structure. However, the goal is to define as much nonlinear effects as possible in order to be prepared to assemble an adequate reduced order model of Structuresthe structure excited by new earthquake excitations.

The transformation matrix $\Phi_{\mathrm{p}}$, containing the POD modes is calculated from the snapshot matrix by applying the SVD algorithm. Afterwards the transformation into the reduced subspace is performed in the same manner, if the classical method of modal truncation would be applied to a linear system. The low-order set of equations of motion is then

$$
\widetilde{\mathbf{M}} \ddot{\mathbf{q}}_{\mathbf{P}}+\widetilde{\mathbf{C}} \dot{\mathbf{q}}_{\mathbf{P}}+\widetilde{\mathbf{R}}=\widetilde{\mathbf{F}}
$$

where $\widetilde{M}=\Phi_{\mathrm{P}}^{\mathrm{T}} \mathbf{M} \Phi_{\mathrm{P}}$ and $\widetilde{\mathrm{C}}=\boldsymbol{\Phi}_{\mathrm{P}}^{\mathbf{T}} \mathbf{C} \boldsymbol{\Phi}_{\mathbf{P}}$ are mass- and stiffness matrices and $\widetilde{\mathbf{F}}=\boldsymbol{\Phi}_{\mathbf{P}} \mathbf{F}$ is the excitation vector in the POD reduced subspace. The reduced vector of the inner restoring forces $\widetilde{\mathbf{R}}$ is still dependent on the displacement in the physical coordinate $\mathbf{x}$,

$$
\widetilde{\mathbf{R}}=\boldsymbol{\Phi}_{\mathbf{P}}^{\mathbf{T}} \mathbf{R}\left(\boldsymbol{\Phi}_{\mathbf{P}} \mathbf{q}_{\mathbf{P}}\right)=\Phi_{\mathbf{P}}^{\mathbf{T}} \mathbf{R}(x) .
$$

Consequently, the vector of the inner restoring forces $\mathbf{R}(\mathbf{x})$ has to be evaluated from the physical model in the full-order coordinates in every calculation time step. Furthermore, the equations of motion in the reduced-order set are not decoupled and have to be solved numerically. Finally, after the time integration procedure is conducted, the solution vector $\mathbf{q}_{\mathbf{P}}$ dependent on time is transformed back into the physical coordinate $\mathbf{x}$. A visualization of this approach is depicted in Figure 3.

The first remarkable advantage of this procedure is not only, as the matter of fact, the lowdimensional matrix operations, but more importantly is the substantial numerical benefit when applying an explicit time integration scheme such as the central difference method. The critical time step $\Delta t_{c r}$, which is $\frac{2 \pi}{\omega_{n}}$, is inversely proportional to the highest eigenfrequency $\omega_{n}$. Consequently, for finely meshed structures a large number of loops has to be executed in order to 
perform the full time integration procedure to solve the equation of motion (1). On the contrary, the critical time step of the POD reduced system described in equation (15) is much larger and, therefore, the number of calculation time steps is much smaller, which requires a fraction of computational effort compared to full-order system. These numerical issues are discussed in a similar manner by Gutierrez and Zaldivar [30] applied to modal truncation. For the numerical benefit of the combination of the POD with explicit numeric time integration, the reader is referred to Bamer and Bucher [18]. The second big advantage of the new strategy is that the actual time consuming process, which is the evaluation of the snapshot matrix, is only executed once at the beginning of the whole calculation procedure. This a priori assumption of nonlinear mode patterns makes sense if the excitations show physical "similarities", which is the case in earthquake analysis, where a considerably small number of lower modes is mainly affected.

\section{Practical application}

In addition to dealing with the development of the introduced POD-based MOR approach, it is within this section to represent the application of the new proposed MOR strategy on a realistic example. For this purpose, a dynamic structural model of a medical complex, according to its constructional plan, was derived. A schematic three-dimensional sketch of the building is depicted in Figure 4.

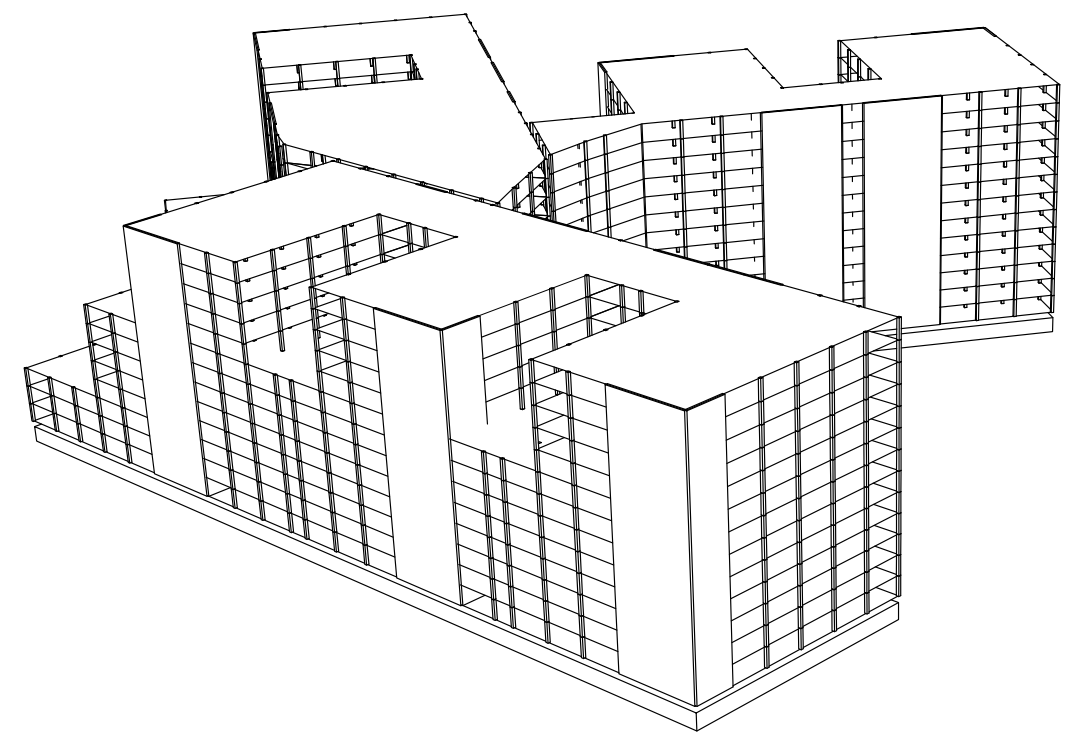

Figure 4: Three-dimensional visualization of the building construction

As shown in Figure 4, the building structure exhibits complex geometries. As a result, it seems to make sense to discretize the geometry by a finite element model in order to capture the main dynamic specifications.

If such a structure with medical function is located in an earthquake prone region, one way to improve its seismic performance can be realized through base isolation by means of frictional pendulum bearings. Consequently, the analytical simulations demand large computational time and storage due to the presence of nonlinearity imposed by those frictional isolators. In the following, firstly, the structural system specifications and implementation of frictional bearings 
are presented. Then, the displacement responses to a set of six earthquake events are evaluated. The numerical evaluations compare the new introduced strategy, as an alternative means, with the iterative Newmark integration scheme, which is known as an efficient and exact method.

\subsection{Structural system and model specifications}

The building structure consists of three wings, referred to as wing I, II and III. Figure 5 shows a schematic sketch of the ground plan of the building containing the basic dimensions. The floor slabs of each wing are separate from the others except for the basement slab, which is indiscrete over all three wings. This means that all three wings are coupled through this slab and they work all together during earthquake excitations. However, the distance between the wings, which are connected by the basement slab, is about 1.5 meters, consequently, contact problems induced by ground motion are not considered in the computations.

The grid indicates the location of the columns and the binding beams, and the red lines indicate the location of the shear walls, which are responsible for the lateral reinforcement. The regular distance between the columns is $6.5[\mathrm{~m}]$. The building structure has three stories below the ground level, while the highest parts of the building above ground level have 13 stories and the remaining parts have eight stories including the basement levels. Therefore, the plan of the structure is irregular along its height along with the irregularities in the horizontal area. The dashed lines define the area, where the building is only located below the ground. The height of one story is three meters; this leads to a total construction height of 42 meters.

Below the three stories at the basement level, there is the indiscrete slab on the top of the isolators at level of $-9.00[\mathrm{~m}]$. Below this slab, along each of the columns, a single friction pendulum (FP) bearing system is attached. Figure 6 depicts a part of the cross section A-A of the basement level shown in Figure 5. The horizontal diameter of the FP system is 2.00 meter. Thus, the dimension of the quadratic cross section of the columns in the basement and FP story is $2.00 \times 2.00\left[\mathrm{~m}^{2}\right]$, while in the remaining stories the columns are modeled as quadratic cross sections with the dimensions $0.40 \times 0.40\left[\mathrm{~m}^{2}\right]$. All FP bearings have the same radius of the concave surface, which is equal to 3.00 meters.

A representative full-scale finite element model of the building structure was created in the software package slangTNG [39]. The shear walls and slabs were modeled by shell/plate elements and the columns and beams by beam elements. A linear elastic material was considered for the modeling purpose (Young's modulus $E=3.5 \cdot 10^{10}\left[\frac{\mathrm{N}}{\mathrm{m}^{2}}\right]$, Poisson's ratio $\nu=0.3[-]$, density $\rho=2500\left[\frac{\mathrm{kg}}{\mathrm{m}^{3}}\right]$ ). Nonlinear FP elements, whose implementation in slangTNG is presented in section 5.2, are assigned below the lowest basement plate of the structure. The total number of degrees of freedom is 33000 .

\subsection{Dynamic model of the frictional pendulum element}

This is to present how the frictional pendulum (FP) element in the finite element model of the structure behaves. The geometrical diagram of the FP element, which is realized as a spherical shell, is defined in Figure 7. As depicted, $R$ denotes the radius of the concave spherical surface and the origin of the local coordinate system is chosen to be in the center of the sphere. The position vector of the slider is described by $\mathbf{U}=[u, v, w]^{T}$. Since the desired behavior of the FP element is an in-plane elasto-plastic bidirectional action, the change of the vertical position $w$ can be neglected. Accordingly, the displacement of the FP element is reduced to an in-plane motion defined only by the components $u$ and $v$, i.e. $\mathbf{U}=[u, v]^{T}$. This simplification makes sense as the radius $R$ is much larger relative to the horizontal displacement 


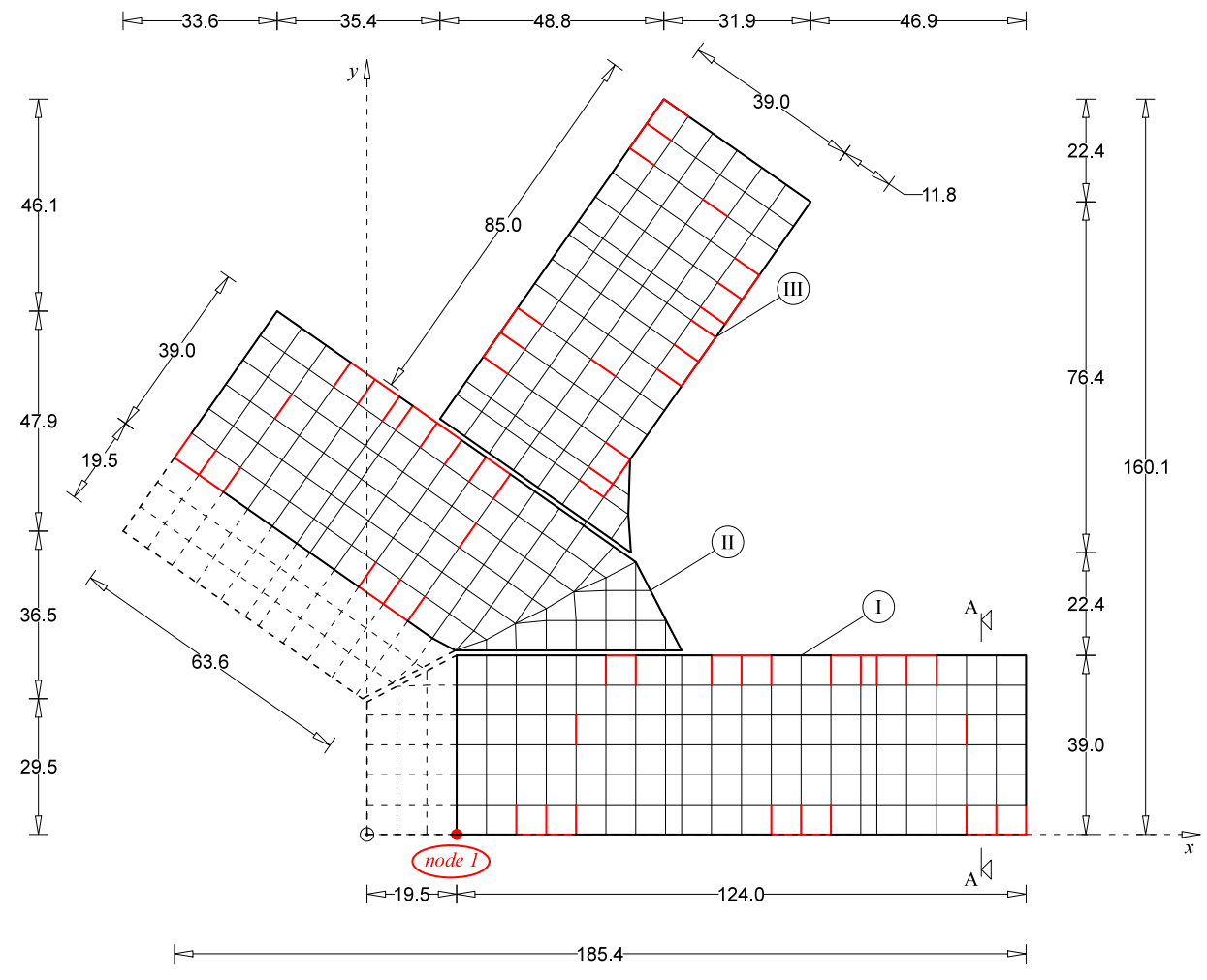

Figure 5: Schematic ground plan, building construction (units in meters), output node $1[19.5,0.0,-9.5]^{T}[m]$

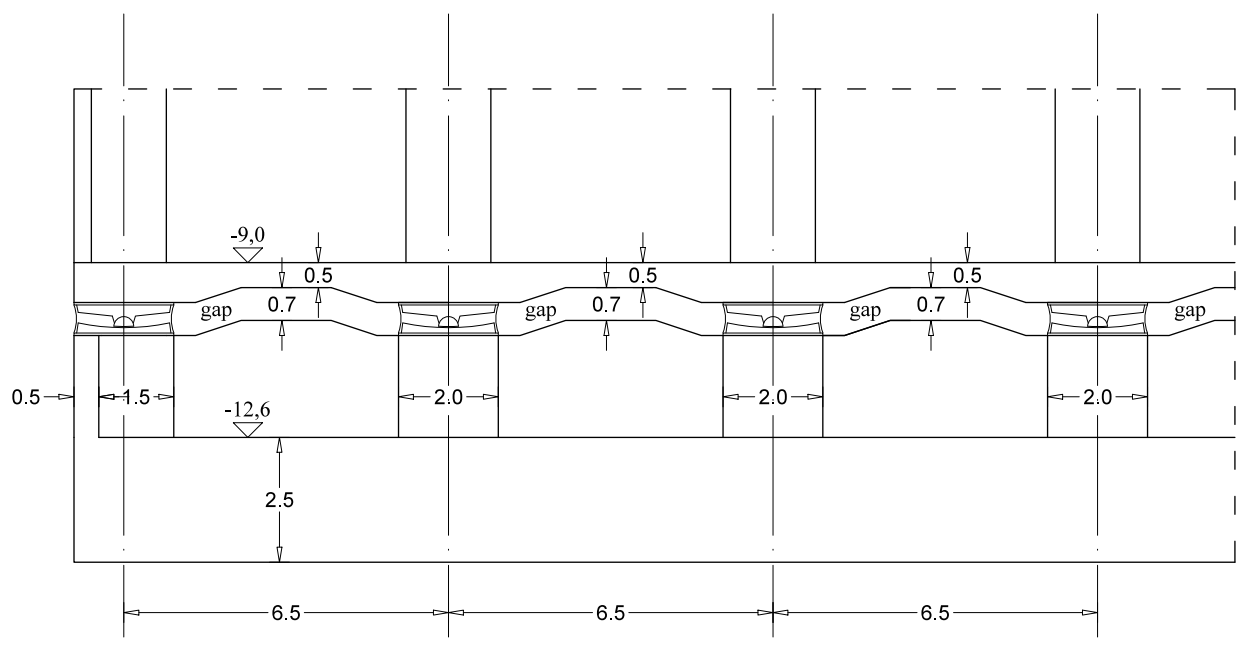

Figure 6: A schematic cutout of the vertical section A-A of the basement levels presented in Figure 5 (units in meters) 
$|\mathbf{U}| \approx \sqrt{u^{2}+v^{2}}$. The equivalent representation of such an element together with the acting forces on it is represented in Figure 8.

The horizontal force equilibrium of the dynamical system is

$$
\mathbf{F}_{\mathrm{Fr}}+\mathbf{F}_{\mathbf{k}}=\mathbf{F}_{\mathrm{ex}}
$$

where $\mathbf{F}_{\mathbf{F r}}$ and $\mathbf{F}_{\mathbf{k}}$ are the elasto-plastic frictional- and centring force and $\mathbf{F}_{\mathbf{e x}}=\left[F_{x}, F_{y}\right]^{T}$ accounts for the interacting horizontal force, which couples the FP element to the super structure. Following, the force equilibrium is split into two parts as two dynamic situations can occur: situation stick and situation slide. The force equilibrium during the situation stick yields to

$$
\mathbf{F}_{\mathbf{e x}}=\underbrace{k_{1}\left\{\begin{array}{c}
u \\
v
\end{array}\right\}}_{\mathbf{F}_{\mathbf{k}}}+\underbrace{k_{2}\left\{\begin{array}{c}
\Delta u \\
\Delta v
\end{array}\right\}}_{\mathbf{F}_{\mathbf{F r}}} \text { if } \quad\left|\mathbf{F}_{\mathbf{e x}}-\mathbf{F}_{\mathbf{k}}\right|<\mu N .
$$

This relation renders a linearly-elastic system, where the friction coefficient $\mu$ must be a value between 0 and 1 (about 0.04 for a realistic implementation of the friction bearing) and the normal contact force $N$ acts orthogonal to the contact area of the slider and the concave surface. The vector $\Delta \mathbf{U}=[\Delta u, \Delta v]^{T}$ defines the radial distance with respect to the current sticking point of the slider if the sticking condition is true.

During the situation slide the FP element is described by the following horizontal force equilibrium

$$
\mathbf{F}_{\mathbf{e x}}=\underbrace{k_{1}\left\{\begin{array}{c}
u \\
v
\end{array}\right\}}_{\mathbf{F}_{\mathbf{k}}}+\underbrace{\frac{\mu N}{|\dot{\mathbf{U}}|}\left\{\begin{array}{c}
\dot{u} \\
\dot{v}
\end{array}\right\}}_{\mathbf{F}_{\mathbf{F r}}} \text { if } \quad\left|\mathbf{F}_{\mathbf{e x}}-\mathbf{F}_{\mathbf{k}}\right| \geq \mu N,
$$

where $\dot{\mathbf{U}}=[\dot{u}, \dot{v}]^{T}$ is the velocity vector.

In both relations, i.e. Eq. (18) and Eq. (19), the centring force $\left|\mathbf{F}_{\mathbf{k}}\right|=k_{1} r=k_{1} \sqrt{u^{2}+v^{2}}$ acts linearly orthogonal to the vertical axis through the deepest point of the surface and the center of the sphere. The fact that the centring force is linear indicates that the spherical shell of the real system is approximated by the paraboloid, whose potential energy increases with $\frac{W}{R} r$ in radial distance from the deepest point, i.e. the stiffness is inversely proportional to the radius of the sphere $k_{1}=\frac{W}{R}$.

The frictional force $\mathbf{F}_{\mathbf{F r}}$ is modeled either linearly elastic or elastic-perfectly plastic as presented in Eqs. (18) and (19), respectively. Note that the force corresponding to $\Delta U$ accounts for the elastic behavior of the bearing coating material, in a small elastic range (situation stick, Eq. (18)) and acts towards the current location of the slider (not the center of the concave sphere). Generally, the implementation of a realistic model requires $k_{2}$ to be much larger than $k_{1}$, i.e. $k_{2} \gg k_{1}$. During the situation slide, the frictional force acts in opposite direction to the velocity with the magnitude (perfectly plastic) $\mu N$. This is discussed in Eq. (19). Another point regarding Eq. (18) is that the reacting force $N$ is assumed to be constant throughout the calculation procedure. This is justified by the following reasons: Firstly, just $x$ and $y$ components of the exciting ground motion are taken into account for the computations. Secondly, the motion has already been simplified to be planar and therefore no additional force component due to vertical motion is generated. Finally, in our preliminary analysis, the uplift force on the isolator slap was observed to be extremely small in comparison with the downward force due 


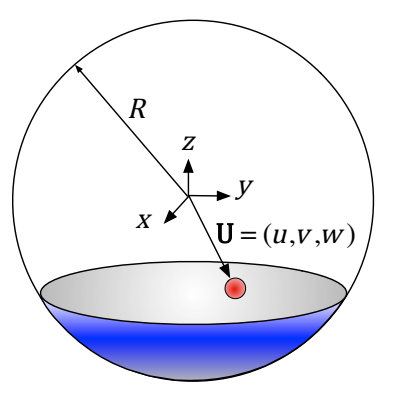

Figure 7: Geometric definitions of the FP element

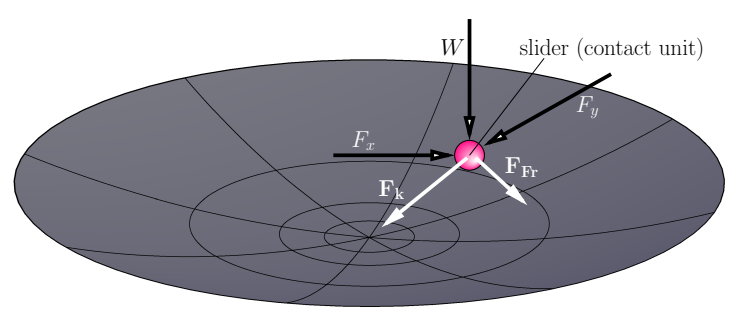

Figure 8: Internal specifications of the FP element; $F_{x}$, $F_{y}, N$, recentering force $\mathbf{F}_{\mathbf{k}}$, friction force $F_{F r}$

\begin{tabular}{cccccccc}
\hline Event & year & location & $n_{t}$ & $T$ & $d$ & $M$ & PGA \\
\hline Bam & 2003 & Iran & 1995 & 19.95 & - & 6.6 & 7.16 \\
Imperial Valley & 1979 & California / Huston Road & 3905 & 39.05 & 10 & 6.5 & 4.79 \\
Landers & 1992 & California / Barstow & 4932 & 49.32 & 36 & 7.3 & 4.13 \\
Loma Prieta & 1989 & California / Gilroy & 2507 & 25.07 & 12 & 7.0 & 9.51 \\
North Palm Springs & 1986 & California / Palm Springs & 6009 & 60.09 & 6.7 & 6.0 & 9.99 \\
Northridge Rinaldi & 1994 & California / Newhall & 1200 & 12.00 & 6.7 & 6.7 & 5.23 \\
\hline
\end{tabular}

Table 1: Earthquake excitation list; $n_{t}[-]$ number of time steps, $T[s]$ duration of the record, $d[k m]$ distance from epicenter, $M$ moment magnitude, $\mathrm{PGA}\left[\mathrm{m} / \mathrm{s}^{2}\right]$ peak ground acceleration

to the weight of the structure. Considering the above-mentioned fact together with the force diagram given in in Figure 8, follows that the normal contact force $N$ is approximately constant and equal to the weight induced force of the super structure, $W$, i.e, $N=W$ in Eq. (19).

The FP bearing element governed by Eqs. (17) to (19) has been implemented in the software package slangTNG [39]. For a comparable study on this implemented friction pendulum system, the experimental work of Mosqueda et al. [40] is suggested. For additional information about friction pendulum systems the reader is referred to the relevant literature (e.g. [33], [34], [35], [36], [37] and [38]). More detailed examination of this topic would lead beyond the scope of this paper, which should focus more on the methodical extension of the new MOR strategy as well as the application on a complex realistic system.

\subsection{Numerical evaluation}

The evaluation of the introduced MOR strategy is dealt with displacement response calculations of the building structure to six different earthquake excitations. The earthquake records are applied in fault-parallel and fault-normal directions. The excitation set includes the Bam earthquake (2003) in Iran and the following five representative events in California, US: Northridge Rinaldi (1994), Imperial Valley (1979), Landers (1992), Loma Prieta (1989), North Palm Springs (1986). Table 1 presents a list of the events taken from the Pacific Earthquake Engineering Research Center (PEER) [31]. Fault-parallel is defined in $x$ - and fault-normal in $y$ direction. Concerning the Bam event only a one-dimensional record was accessible, therefore, an excitation angle of 30 degrees with respect to the $x$-axis was chosen. Calculation outputs are presented including the displacement translational response, namely the degree of freedom $x$ of the red marked node in Figure 5. This is the horizontal component of response in $x$-direction of the slider of the FP element. Additionally, the response regarding in-plane motion of the slider 
in $x$ - and $y$-direction are provided. The output node is called "node 1" and has the coordinates $[19.5,0.0,-9.5]^{T}[m]$, this is depicted in Figure 5. This node defines the location of a moving friction pendulum, it therefore shows directly the nonlinear response behavior of the system.

The method, according to Section 4, begins with the earthquake dynamic analysis of the structure under representative excitation. Therefore, the response $\mathbf{X}_{B a m}=\left[\mathbf{x}_{\mathbf{1}}, \mathbf{x}_{\mathbf{2}}, \ldots, \mathbf{x}_{\mathbf{m}}\right]$ of the full system to the Bam earthquake is evaluated. This full system response is evaluated applying the Newmark method. The response history in $x$-direction of node 1 (output node) is depicted in the left subplot of Figure 9. The motion of the slider is shown in the right subplot of Figure 9.
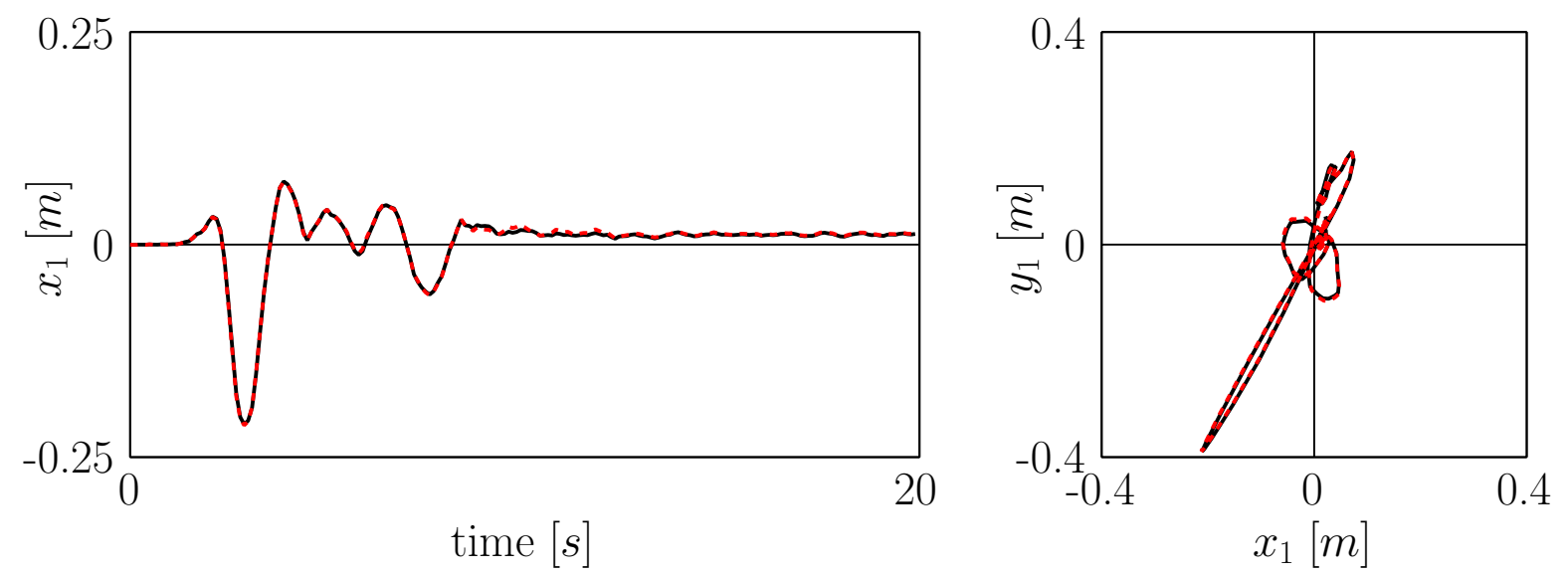

Figure 9: Response functions to the Bam earthquake excitation, full Newmark response and reduced POD response; left subplot: displacement $x$ of node 1 dependent on time; right subplot: movement of the slider, displacement $x$ and $y$ of node 1

The snapshot matrix $\mathbf{X}_{\mathbf{S}}$ is assembled by taking into account 400 snapshots in equidistant time intervals spread over the whole displacement response history to the Bam earthquake excitation. It is essential to capture the main deformation behavior of the system in this stage, otherwise certain deformation states cannot be constituted by the set of linearly independent vectors (c.f. Figure 2), i.e. the sliding process, as can be seen in Figure 9, has to be recorded in the snapshot matrix. Afterwards, the evaluation of the left singular vectors of the snapshot matrix leads to the POD modes and its singular values to the POD values in descending order. The sum of all POD values define the total energy content of the snapshot matrix. Hence, the number of POD modes that have to be taken into account in order to capture 99, 99 percent of the total energy was evaluated to be 31 . A plot of the first 31 singular values (POD values) dependent on the corresponding energy content is shown in Figure 10.

The physical equation of motion is now transformed into the 31-dimensional POD subspace and integrated over the whole time history by application of the explicit central difference scheme. Transformation back into the physical coordinate $\mathbf{x}$ produces the POD-based response of the structure. The integration time step for the reduced time integration is $5 \cdot 10^{-3}$ seconds, which is equivalent to the time step of the Newmark integration scheme, which is applied for the calculation of the exact response. In the response analysis of the reduced order system via central difference no iteration is needed and only a fractional amount of storage compared to the full Newmark method is required. Note that the critical time step of the central difference scheme for the full system is $10^{-7}$ seconds. Therefore, concerning the basic central difference method, an immense speed-up factor of $10^{4}$ is achieved. Within the presented examples generally a min- 


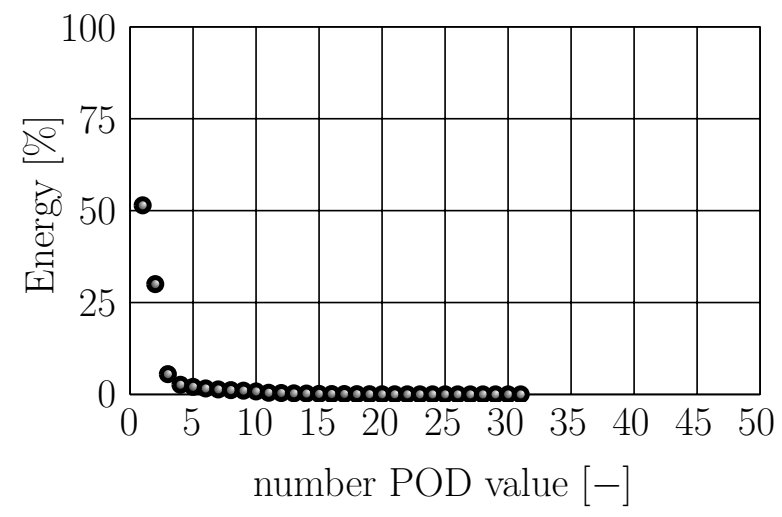

Figure 10: Number of POD values (singular values)

imum speed up factor of about 2 compared to the Newmark algorithm is achieved. However, it has to be added that for this type of nonlinearity, the Newmark integration scheme appears to have a relative slow convergence rate. As a consequence, the computational time by applying the Newmark method can exceed to a period of time, which is comparable if the full central difference integration scheme is applied or even to infinite period of time, if there is no convergence. Following, the new integration strategy gains the benefit of both of the two algorithms, i.e. central difference and Newmark, which is stability without requiring iteration algorithms. The red dashed line in Figure 9 (response to the Bam excitation) shows the response obtained by the new POD strategy, which approximates the full Newmark response accurately. This is not surprising as the snapshots are taken in equidistant time intervals spread over this whole response history. The derived POD transformation matrix, which is the set of POD modes, is now applied to reduce the order of the set of equations for the structure excited by the rest of the presented earthquake events presented in Table 1. It means that the time integration in the full (physical) space no longer has to be performed. As for the next transformations into the POD space only one transformation matrix is applied. This strategy is here called universal POD method. Now this approach unveils its similarities to the method of modal truncation, which is mainly applicable to linear systems. Figures 11, 12, 13, 14 and 15 compare the displacement responses applying the new MOR strategy and the Newmark scheme.

Accurate approximations are achieved by means of the proposed MOR strategy. The response to the Imperial Valley earthquake (Figure 11), the Landers earthquake (Figure 12) and the North Palm Springs earthquake (Figure 14) were highly accurate, when the proposed MOR strategy was applied. Concerning the response functions of the Loma Prieta (Figure 13) and the North Palm Springs excitation (Figure 15) small variations concerning the full and the reduced solutions can be observed. This is because the responses to those excitations include deformation states that are not captured in the snapshot matrix and, hence, not in the universal POD modes (POD transformation matrix) and thus cannot assemble the exact response history.

\section{Conclusions}

In this paper, a model order reduction (MOR) strategy, which is applicable to the dynamic response analysis of linear and nonlinear structural systems was presented. Usually, the analysis of building structures with complex geometries makes the engineer to create a finite element model with a large number of degrees of freedom, which is associated with computational effort in the response analysis. Therefore, the goal of this paper is to provide a new practical 

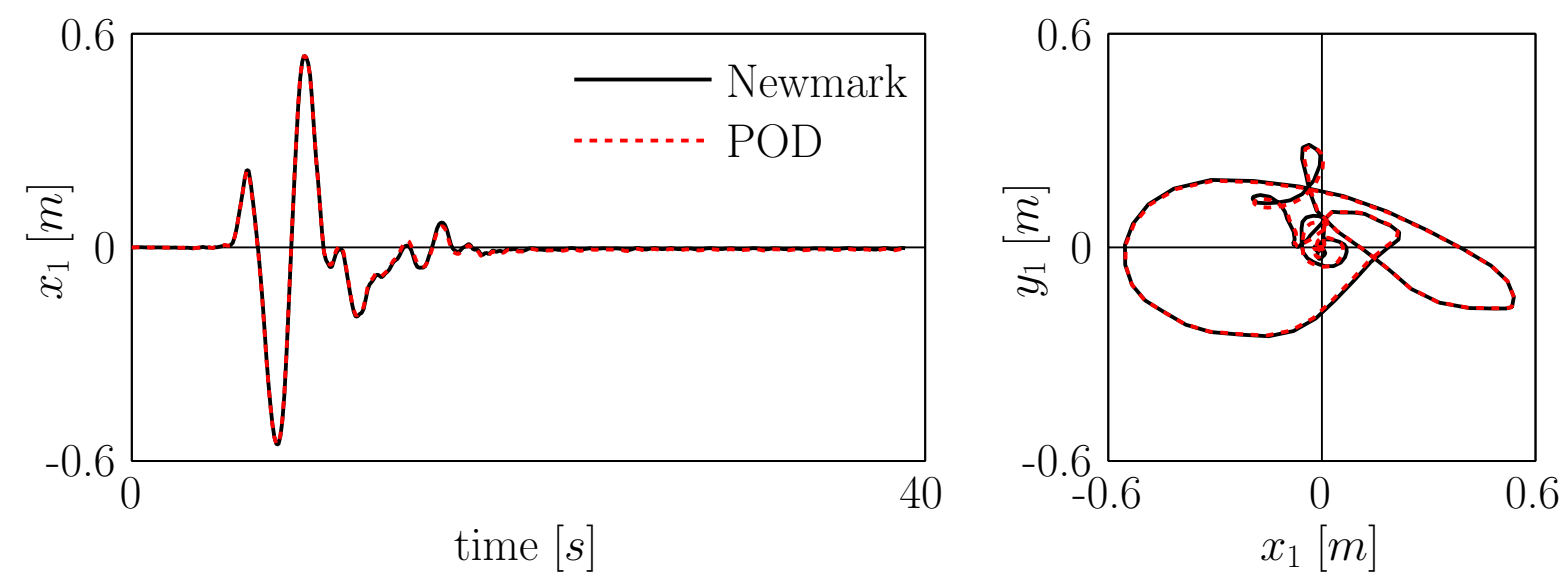

Figure 11: Response functions to the Imperial Valley excitation, full Newmark response and universal reduced POD response; left subplot: displacement $x$ of node 1 dependent on time; right subplot: movement of the slider, displacement $x$ and $y$ of node 1
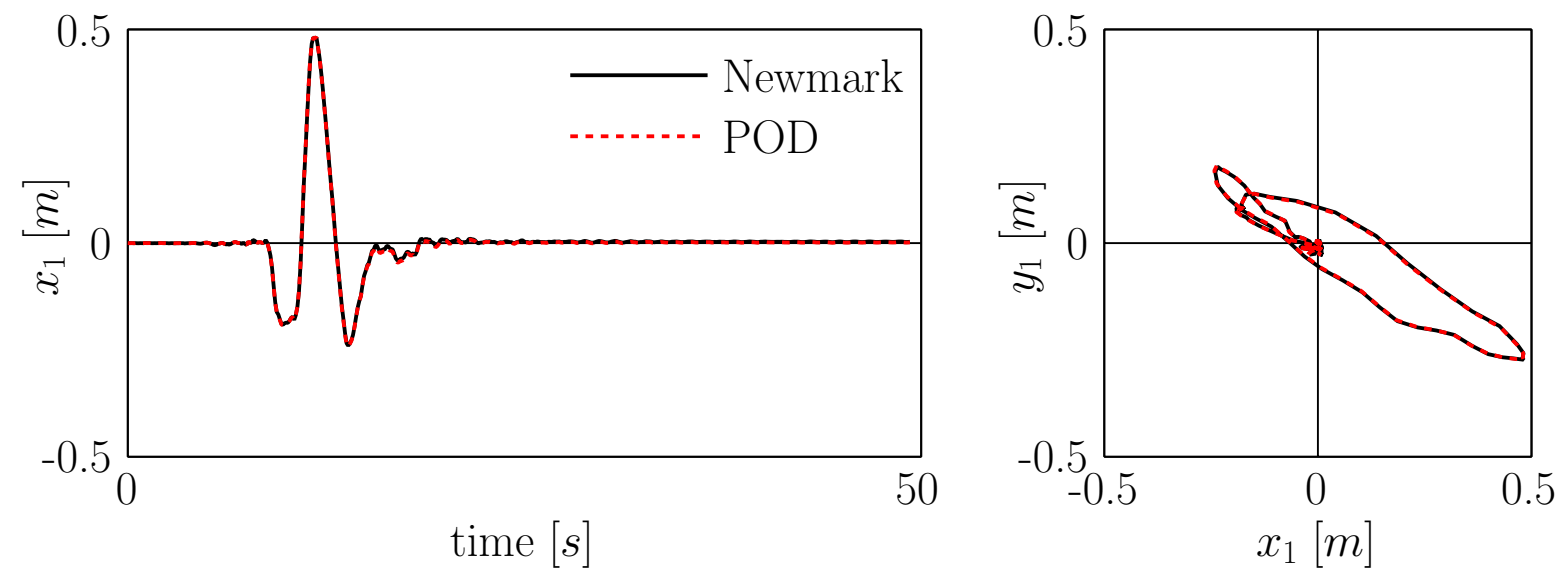

Figure 12: Response functions to the Landers excitation, full Newmark response and universal reduced POD response; left subplot: displacement $x$ of node 1 dependent on time; right subplot: movement of the slider, displacement $x$ and $y$ of node 1 

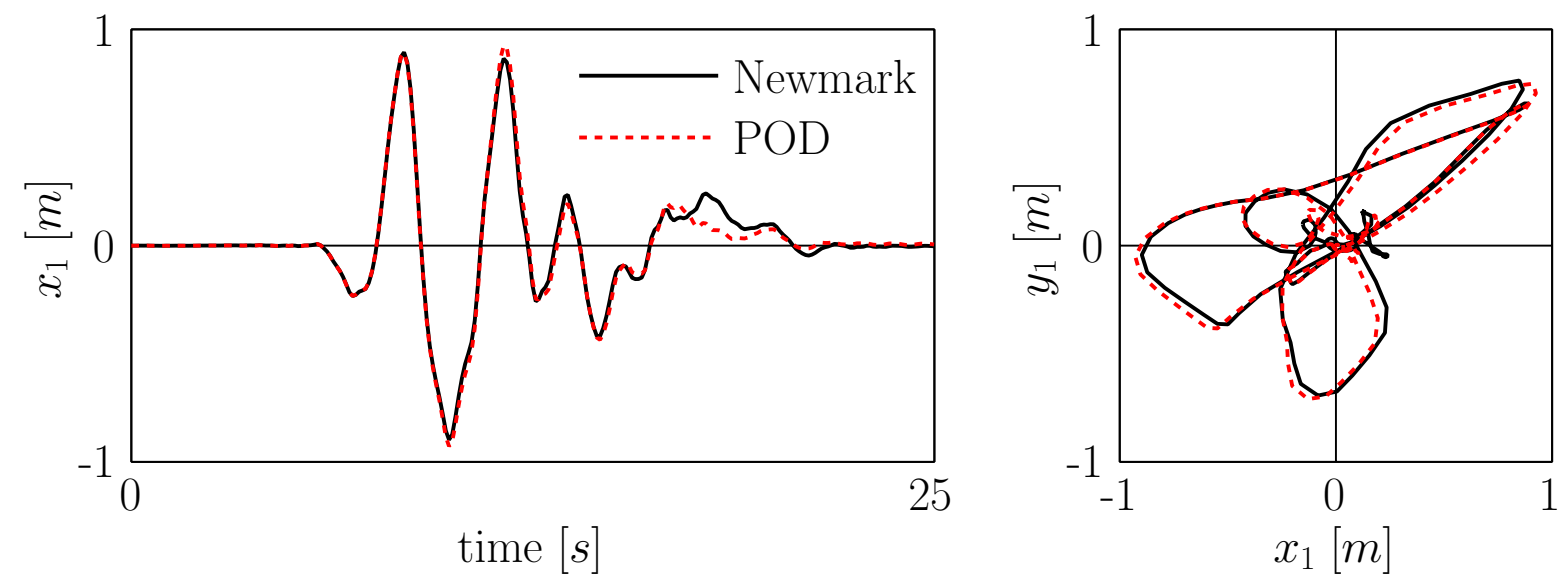

Figure 13: Response functions to the Loma Prieta excitation, full Newmark response and universal reduced POD response; left subplot: displacement $x$ of node 1 dependent on time; right subplot: movement of the slider, displacement $x$ and $y$ of node 1
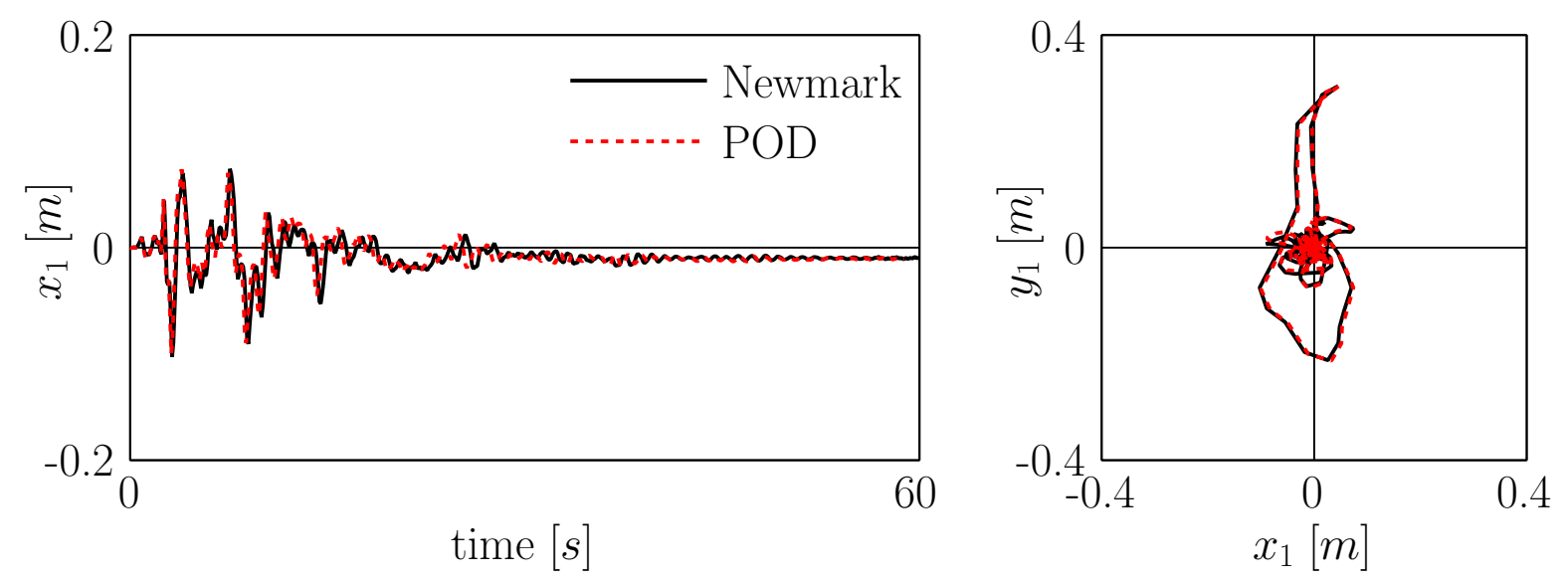

Figure 14: Response functions to the North Palm Springs excitation, full Newmark response and universal reduced POD response; left subplot: displacement $x$ of node 1 dependent on time; right subplot: movement of the slider, displacement $x$ and $y$ of node 1 

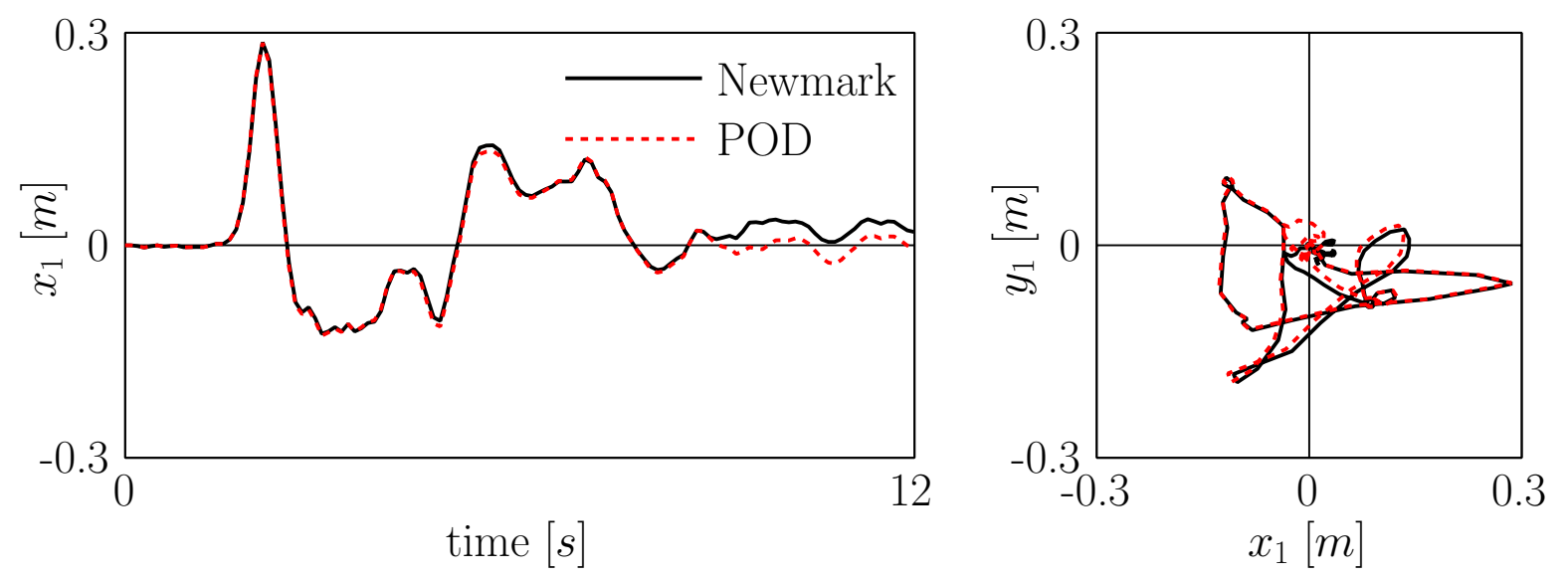

Figure 15: Response functions to the Northridge excitation, full Newmark response and universal reduced POD response; left subplot: displacement $x$ of node 1 dependent on time; right subplot: movement of the slider, displacement $x$ and $y$ of node 1

model order reduction strategy that is simple in application, but also very effective even in the presence of nonlinearities for problems in the field of earthquake engineering and structural dynamics. This strategy is extended based on the proper orthogonal decomposition (POD) method to derive a proper transformation matrix in order to transform the nonlinear systems into another low-dimensional subspace, which demands considerably less computational effort for the response calculation. Once the transformation matrix is derived, the approach of the strategy is similar to the method of modal truncation for linear systems.

In addition to the development of the MOR strategy, its application for the response calculation of a realistic numerical nonlinear example is demonstrated. The example is the displacement response calculation of a building structure serving as a medical complex, which is base-isolated by friction pendulum bearing systems excited by six earthquake excitations. In order to evaluate the accuracy of the introduced approach, the exact structural responses were also calculated by the iterative Newmark method in the full order (physical) coordinates. Numerical evaluations show that accurate approximations can be achieved if nonlinear response patterns of the structure are already captured in the POD snapshots to extract the transformation matrix. The advantage of this strategy is that obviously the transformation matrix is derived just once and it can be used for response calculation of the structure under different earthquake excitations.

Another substantial advantage of the introduced MOR concerns the speed of the response calculations. Firstly, compared to the basic central difference algorithm, the new introduced strategy has a much larger critical time step. Secondly, compared to the Newmark method, which allows usually larger time steps, no iteration procedure is required.

\section{Acknowledgements}

The authors would like to acknowledge financial support from the Austrian Science Funds (FWF) as part of the Vienna Doctoral Programme on Water Resource Systems (DK-plus W1219N22). This research has also been supported by Stiftung Maurer Söhne (Forschungsförderung Technische Dynamik), which is gratefully acknowledged by the authors. Special thanks of the authors is dedicated to Professor Bernd Markert, head of the Institute of General Mechanics at the RWTH Aachen University, for his critical feedbacks and constant supports. 


\section{REFERENCES}

[1] K.J. Bathe, Finite Element Procedures. Prentice Hall, 1995.

[2] Z.Q. Qu, Model order reduction techniques with application in finite element analysis. Springer London Limited, 2004.

[3] G. Rega, H Troger, Dimension Reduction of Dynamical Systems: Methods, Models, Applications. Nonlinear Dynamics, 41, 1-15, 2005.

[4] P. Koutsovasilis, M. Beitelschmidt, Comparison of model reduction techniques for large mechanical sytems, Multibody System Dynamics, 20, 111-128, 2008.

[5] H.A. Schilders, H.A. Vorst, J. Rommes, Model Order Reducion, Theory, Research Aspects and Application. Springer, 2008.

[6] D. Kosambi, Statistics in function space, Journal of the Indian Mathematical Society, 1943.

[7] K. Karhunen, Über lineare Methoden in der Wahrscheinlichkeitsrechnung, PhD thesis, University of Helsinki, 1947.

[8] M. Loeve, Fonctions al Eatoire de second ordre, Revue Scientifique, 48, 195-206, 1946.

[9] J.P. Cusumano, M.T. Sharkady, B.W. Kimble, Spatial coherence measurements of a chaotic flexible-beam impact oscillator, American Society of Mechanical Engineers, Aerospace Division (Publication) AD, 33, 13-22, 1993.

[10] B.F. Feeny, R. Kappagantu, On the physical interpretation of proper orthogonal modes in vibrations, Journal of Sound and Vibration, 211, 607-616, 1998.

[11] R. Kappagantu, B.F. Feeny, An "optimal" modal reduction of a system with frictional excitation, Journal of Sound and Vibration, 224, 863-877, 1999.

[12] R. Kappagantu, B.F. Feeny, Part 1: Dynamical Characterization of a frictionally excited beam, Nonlinear Dynamics, 22, 317-333, 2000.

[13] R. Kappagantu, B.F. Feeny, Part 2: Proper Orthogonal Modal Modeling of a Frictionally Excited Beam, Nonlinear Dynamics, 23, 1-11, 2000.

[14] Y.C. Liang, H.P. Lee, S.P. Lim, W.Z. Lin, K.H. Lee, C.G. Wu, Proper Orthogonal Decomposition and its Applications - Part 1: Theory, Journal of Sound and Vibration, 252, 527-544, 2002.

[15] G. Kerschen, J.C. Golivani, Physical interpretation of the proper orthogonal modes using the singular value decomposition, Journal of Sound and Vibration, 249, 849-865, 2002.

[16] G. Kerschen, J.C. Golivani, A model updating strategy of non-linear vibrating structures, International Journal of Numerical Methods in Engineering, 60, 2147-2164, 2003.

[17] F. Tubino, L. Carassale, G. Solari, Seismic response of multi-supported structures by proper orthogonal decomposition, Earthquake Engineering and Structural Dynamics, 32, 1639-1654, 2003. 
[18] F. Bamer, C. Bucher, Application of the proper orthogonal decomposition for linear and nonlinear structures under transient excitation, Acta Mechanica, 223, 2549-2563, 2012.

[19] P. Koutsovasilis, M. Beitelschmidt, Comparison of model reduction techniques for large mechanical sytems, Multibody System Dynamics, 20, 111-128, 2008.

[20] A.K. Chopra, Dynamics of Structures, Theory and Applications to Earthquake Engineering. Prentice Hall, 2001.

[21] A. Chatterjee, An introduction to the proper orthogonal decomposition, Current Science, 78, 808-817, 2000.

[22] S. Volkwein, Model reduction using proper orthogonal decomposition. Karl-FranzensUniversität Graz, Institut für Mathematik und Wissenschaftliches Rechnen, 2008.

[23] S. Volkwein, Proper Orthogonal Decomposition and Singular Value Decomposition. Karl-Franzens-Universität Graz, Institut für Mathematik und Wissenschaftliches Rechnen, 2008.

[24] P. Holmes, J.L. Lumley, G. Berkooz, Turbulence, Coherent Structures, Dynamical Systems and Symmetry. Cambridge University Press, 1996.

[25] S. Han, B. Feeny, Application of proper orthogonal decomposition to structural vibration analysis, Mechanical Systems and Signal Processing, 17, 989-1001, 2003.

[26] G. Kerschen, J.C. Golinval, A.F. Vakakis, L.A. Bergman, The Method of Proper Orthogonal Decomposition for Dynamical Characterization and Order Reduction of Mechanical Systems: An Overview, Nonlinear Dynamics, 41, 147-169, 2005.

[27] Z. Qu, Y. Shi, H. Hua, A reduced-order modeling technique for tall buildings with active tunded mass damper, Earthquake Engineering and Structural Dynamics, 30, 349-362, 2001.

[28] A.G. Schemann, H.A. Smith. Vibration control of cable-stayed bridges - part 1: modelling issues, Earthquake Engineering and Structural Dynamics, 27, 811-824, 1998.

[29] E. Gutierrez, J.M. Zaldivar, The application of Karhunen-Loeve, or principal component analysis method, to study the non-linear seismic response of structures, Earthquake Engineering and Structural Dynamics, 29, 1261-1286, 2000.

[30] E. Gutierrez, J.J.L. Cela, Improving explicit time integration by modal truncation techniques, Earthquake Engineering And Structural Dynamics, 27, 1541-1557, 1998.

[31] Pacific Earthquake Engineering Research Center (PEER), Homepage: http://nisee.berkeley.edu, 2015.

[32] C. Bucher, Stabilization of explicit time integration by modal reduction, Trends in computational structural mechanics, 2001.

[33] J. Almazan, J. Llera, J. Inaudi, Modelling aspects of structures isolated with the frictional pendulum system, Earthquake Engineering and Structural Dynamics, 27, 845-867, 1998. 
[34] J. Llera, J. Almazan, An experimental study of nominally symmetric and asymmetric structures isolated with the FPS, Earthquake Engineering and Structural Dynamics, 32, 891-918, 2003.

[35] D. Ordonez, D. Foti, L. Bozzo, Comparative study of the inelastic response of base isolated buildings, Earthquake Engineering and Structural Dynamics, 32, 151-164, 2003.

[36] K. Ryan, A.K. Chopra, Estimating the seismic displacement of friction pendulum isolators based on non-linear response history analysis, Earthquake Engineering and Structural Dynamics, 33, 359-373, 2004.

[37] T. Ray, A. Sarlis, A. Reinhorn, M. Constantinou, Hysteretic models for sliding bearings with varying frictional force, Earthquake Engineering and Structural Dynamics, 42, 2341-2360, 2013.

[38] C. Bucher, Probability-based optimal design of friction-based seismic isolation devices, Structural Safety, 31, 500-507, 2009.

[39] C. Bucher, Open source structural dynamics and FE software (slangTNG), Homepage: http://info.tuwien.ac.at/bucher/Private/Welcome.html, 2015.

[40] G. Mosqueda, A. Whittaker, G. Fenves, Characterization and Modeling of Friction Pendulum Bearings Subjected to Multiple Components of Excitation, Journal of Structural Engineering ASCE, 130, 433-442, 2004. 